\title{
Electron-hole pair effects in methane dissociative chemisorption on $\mathrm{Ni}(111)$
}

Cite as: J. Chem. Phys. 145, 044704 (2016); https://doi.org/10.1063/1.4959288

Submitted: 11 May 2016 . Accepted: 11 July 2016 . Published Online: 26 July 2016

Xuan Luo, Bin Jiang, J. Iñaki Juaristi, Maite Alducin, and Hua Guo
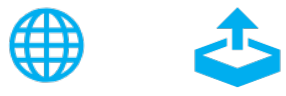

\section{ARTICLES YOU MAY BE INTERESTED IN}

Effects of surface motion and electron-hole pair excitations in $\mathrm{CO}_{2}$ dissociation and scattering on $\mathrm{Ni}(100)$

The Journal of Chemical Physics 148, 174702 (2018); https://doi.org/10.1063/1.5025029

Dynamics in reactions on metal surfaces: A theoretical perspective

The Journal of Chemical Physics 150, 180901 (2019); https://doi.org/10.1063/1.5096869

Ab initio molecular dynamics study of the Eley-Rideal reaction of $\mathrm{H}+\mathrm{Cl}-\mathrm{Au}(111) \rightarrow \mathrm{HCl}+$

$\mathrm{Au}(111):$ Impact of energy dissipation to surface phonons and electron-hole pairs

The Journal of Chemical Physics 148, 014702 (2018); https://doi.org/10.1063/1.5016054

Challenge us.

What are your needs for periodic signal detection?

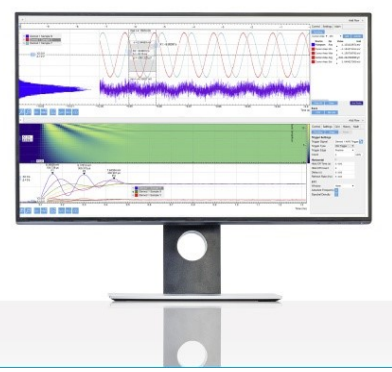




\title{
Electron-hole pair effects in methane dissociative chemisorption on $\mathrm{Ni}(111)$
}

\author{
Xuan Luo, ${ }^{1}$ Bin Jiang, ${ }^{1, a)}$ J. Iñaki Juaristi, ${ }^{2,3,4}$ Maite Alducin, ${ }^{2,3}$ and Hua Guo ${ }^{5}$ \\ ${ }^{1}$ Department of Chemical Physics, University of Science and Technology of China, Hefei, Anhui 230026, China \\ ${ }^{2}$ Centro de Física de Materiales CFM/MPC(CSIC-UPV/EHU), P. Manuel de Lardizabal 5, \\ 20018 San Sebastián, Spain \\ ${ }^{3}$ Donostia International Physics Center DIPC, P. Manuel de Lardizabal 4, 20018 San Sebastián, Spain \\ ${ }^{4}$ Departamento de Física de Materiales, Facultad de Químicas, Universidad del País Vasco (UPV/EHU), \\ Apartado 1072, 20080 San Sebastián, Spain \\ ${ }^{5}$ Department of Chemistry and Chemical Biology, University of New Mexico, Albuquerque, New Mexico 87131, \\ USA
}

(Received 11 May 2016; accepted 11 July 2016; published online 26 July 2016)

\begin{abstract}
The dissociative chemisorption of methane on metal surfaces has attracted much attention in recent years as a prototype of gas-surface reactions in understanding the mode specific and bond selective chemistry. In this work, we systematically investigate the influence of electron-hole pair excitations on the dissociative chemisorption of $\mathrm{CH}_{4} / \mathrm{CH}_{3} \mathrm{D} / \mathrm{CHD}_{3}$ on $\mathrm{Ni}(111)$. The energy dissipation induced by surface electron-hole pair excitations is modeled as a friction force introduced in the generalized Langevin equation, in which the independent atomic friction coefficients are determined within the local-density friction approximation. Quasi-classical trajectory calculations for $\mathrm{CH}_{4} / \mathrm{CH}_{3} \mathrm{D} / \mathrm{CHD}_{3}$ have been carried out on a recently developed twelve-dimensional potential energy surface. Comparing the dissociation probabilities obtained with and without friction, our results clearly indicate that the electron-hole pair effects are generally small, both on absolute reactivity of each vibrational state and on the mode specificity and bond selectivity. Given similar observations in both water and methane dissociation processes, we conclude that electron-hole pair excitations would not play an important role as long as the reaction is direct and the interaction time between the molecule and metal electrons is relatively short. Published by AIP Publishing. [http://dx.doi.org/10.1063/1.4959288]
\end{abstract}

\section{INTRODUCTION}

In the industrial production of syngas via steam reforming, the dissociative chemisorption (DC) of methane on transition-metal catalyst surfaces is the initial and ratelimiting step. ${ }^{1}$ This reaction also serves as a prototype for understanding the dynamics of polyatomic DC at the gas-solid interfaces. ${ }^{2-6}$ State-of-the-art quantum state resolved experiments, mainly from the Beck and Utz groups, have reported significant effects of the translational energy and vibrational excitations on the DC of $\mathrm{CH}_{4}$ and its isotopologues. ${ }^{7-23}$ Special attention has been paid to mode specificity, namely, the different effects of the reactant vibrational modes on the reactivity, and bond selectivity, referring to selective bond breaking. In general, stretching excitations of methane were found to be more effective than their bending counterparts, and in some cases, than the translational energy in promoting the DC. Excitations of certain vibrational modes in partially deuterated methane have also been shown to selectively break bonds. A similar mode specific DC process has been found in another important polyatomic molecule, namely, water. ${ }^{24}$ These observations suggested that all forms of energy are not equal and as a result DC reactions are strongly influenced by dynamics and therefore not describable by statistical models.

\footnotetext{
a) Author to whom correspondence should be addressed. Electronic mail: bjiangch@ustc.edu.cn
}

The pioneering experiments on methane DC offered a challenge and stringent test for theoretical models. Even on a rigid surface, fifteen degrees of freedom (DOFs) are needed to characterize methane, rendering this system very challenging for theoretical characterization. In the last decade, extensive theoretical efforts have been devoted to the understanding of the multi-dimensional interaction potential energy surface (PES) and dissociation dynamics. ${ }^{25-42}$ For example, Jackson and co-workers advanced the use of the reaction path Hamiltonian (RPH) to describe the DC process, ${ }^{29,30}$ in which the vibrational modes perpendicular to the reaction coordinate are approximated as harmonic oscillators. ${ }^{43}$ The same group has also devised effective approximations for including effects of surface impact sites and lattice motion. ${ }^{25,26,28}$ We have approached the problem from a different perspective that is based on constructing a fully coupled high-dimensional PES on the rigid Ni(111) surface by accurately fitting a large number of density functional theory (DFT) energies. ${ }^{31}$ This approach has also been taken by Zhang and co-workers, who have recently reported several high-dimensional PESs for methane DC. . $^{37,38}$ A similar strategy is to parameterize a reaction force field for the same system based on DFT data, as demonstrated by Dong, Busnengo, and co-workers. ${ }^{35,36}$ These global PESs enabled both quantum dynamical (QD) and quasi-classical trajectory (QCT) studies of the DC dynamics. ${ }^{25-32,35,37,38,40-42,44,45}$ On the other hand, $A b$ Initio Molecular Dynamics (AIMD), which requires no pre-calculated PES because the forces 
are obtained on-the-fly from DFT calculations, have been spearheaded by Kroes and co-workers to investigate the $\mathrm{CHD}_{3}$ dissociation on $\mathrm{Ni}(111)$ and $\mathrm{Pt}(111){ }^{33,34,39,41}$ While the quantum effects are treated approximately or ignored altogether, this approach allows the natural inclusion of surface effects. These quantum and classical studies have shed valuable light on the dissociation dynamics and provided the impetus for the establishment of the sudden vector projection (SVP) model $^{46,47}$ to understand the origin of the observed mode specificity and bond selectivity.

Despite the general success of aforementioned classical and quantum dynamical models, a key question remains. All existing theoretical models are based on the adiabatic BornOppenheimer approximation, in which the electronic and nuclear motions are assumed to be separable. However, such an assumption is questionable near a metal surface because the electronic energy levels are near degenerate and thermal electron-hole pairs (EHPs) are prevalent. ${ }^{48}$ Indeed, there is ample evidence of non-adiabaticity near metal surfaces. ${ }^{48-52}$ It is thus conceivable that DC processes can be influenced by surface EHPs, which could dissipate energy in the molecule and compete with the bond cleavage process. In the DC of diatomic molecules like $\mathrm{H}_{2}$ and $\mathrm{N}_{2},{ }^{53-57}$ however, the nonadiabatic effects have been found to be generally very small, although Luntz et al. argued for a more important role of EHPs for $\mathrm{N}_{2} / \mathrm{Ru}(0001) .{ }^{58}$ Very recently, we reported the first study on the effects of EHPs in the DC of a polyatomic molecule, namely, water, on $\mathrm{Ni}(111) .{ }^{59}$ Using a friction model, it was found that the EHPs lead to a small decrease of the dissociation probability, but this decrease is more or less the same for each vibrational state of water, thus statistically having almost no effect on the mode specificity. In the present work, we extend the friction model to study the non-adiabatic effects in the DC of $\mathrm{CH}_{4} / \mathrm{CHD}_{3} / \mathrm{CH}_{3} \mathrm{D}$ on $\mathrm{Ni}(111)$, focusing on the mode specificity and bond selectivity. Sections II-IV are arranged as follows: Section II discusses the friction model and its implementation in quasi-classical trajectories. The results are presented and analyzed in Sec. III. Finally, conclusions are given in Sec. IV.

\section{COMPUTATIONAL DETAILS}

\section{A. Potential energy surface}

In this work, our twelve-dimensional (12D) global PES for describing the DC of methane on a rigid $\mathrm{Ni(111)}$ surface was employed. ${ }^{31}$ Within the flat surface approximation, the lateral coordinates $(X, Y)$ and azimuthal angle $(\phi)$ were neglected, but all other important DOFs in methane DC are included. The PES was fit to over 36000 DFT points computed with the PW91 functional ${ }^{60}$ using the permutationally invariant polynomials method ${ }^{61}$ adapted for gas-surface reactions, ${ }^{62}$ where the projection of the molecular center on the surface is fixed at that of the transition state, very close to the top site. This single-site model is supported by more recent high-dimensional quantum dynamical calculations indicating the dominant role of the top site in the DC of methane. ${ }^{40}$ This PES, which is fast to evaluate and preserves the permutation symmetry of the four $\mathrm{H}$ atoms, has been used in both QD and QCT studies of the methane DC reaction, yielding results that are in good agreement with observations. ${ }^{31,32}$ Although PESs including more DOFs have now been available, ${ }^{35,37,38}$ it is unlikely that the additional DOFs will change the physics in a fundamental way, as the reactivity is dominated by the minimum energy path over the top site, especially at low energies. ${ }^{34,40}$ Quantitatively, the flat surface model imposed here may overestimate the energy dissipation by EHP excitations, because the reactive trajectories that, in the full-dimensional model, deviate from the minimum energy reaction path will have the lower velocities along the reaction coordinate and tend to reduce the friction induced energy dissipation.

\section{B. Friction model}

Following our previous work, ${ }^{59}$ the EHP effects can be modeled as a friction force ${ }^{63,64}$ in the following generalized Langevin equation: ${ }^{65}$

$$
m_{i} \frac{d^{2} \mathbf{R}_{i}}{d t^{2}}=-\frac{\partial V\left(\mathbf{R}_{i}\right)}{\partial \mathbf{R}_{i}}-\eta_{i}\left(\mathbf{R}_{i}\right) \frac{d \mathbf{R}_{i}}{d t}+F_{i}(T),
$$

where $\mathbf{R}_{i}$ and $m_{i}$ are the position and mass of the $i$ th atom in the molecule, $V\left(\mathbf{R}_{i}\right)$ is the adiabatic PES, and $F_{i}(T)$ represents the temperature-dependent random force, which is typically very small at low surface temperatures and was neglected here. The key in Eq. (1) is the dissipation force due to surface EHPs, which is determined by coordinate-dependent atomic friction coefficients $\left(\eta_{i}\left(\mathbf{R}_{i}\right)\right)$ and velocity $\left(\frac{d \mathbf{R}_{i}}{d t}\right)$. We note that the possibility of surface phonon excitations is beyond the scope of this work, but should be included in a complete treatment.

Because of its simplicity and effectiveness, electronic friction models have been successfully used to study a wide range of non-adiabatic processes on metal surfaces, such as atomic and molecular scattering and adsorption, ${ }^{66-76}$ vibrational relaxation of adsorbates, ${ }^{77-81}$ and DC processes. ${ }^{54,55,58,59,69,82}$ In our model, the molecule is assumed as a collection of atoms in the independent-atom approximation (IAA), ${ }^{54}$ further simplifying the calculation. In addition, atomic friction coefficients are obtained with the local-density friction approximation (LDFA) $)^{54,63,78}$ and they depend on the local electronic density in which the atom is embedded. This IAALDFA model has been shown to be reasonably accurate. ${ }^{75,80,81}$

Specifically, the coordinate-dependent friction coefficient for an atom within LDFA is calculated in the free electron gas (FEG) model as ${ }^{54,66,67}$

$$
\eta_{i}=\frac{4 \pi \rho}{k_{F}} \sum_{l=0}^{\infty}(l+1) \sin ^{2}\left[\delta_{l}^{i}\left(k_{F}\right)-\delta_{l+1}^{i}\left(k_{F}\right)\right],
$$

where $k_{F}$ is the Fermi momentum and $\delta_{l}^{i}\left(k_{F}\right)$ are the scattering phase shifts of the Kohn-Sham orbitals at the Fermi level, and $\rho$ is the embedded electron density, which was readily computed on the bare Ni(111) surface using DFT in our earlier work. ${ }^{59}$ The phase shifts in Eq. (2) are usually computed with the local density approximation (LDA) and, therefore, it neglects the possible spin-polarization that may remain in case of open-shell atoms embedded in a low-density FEG. This was also the case of the friction coefficients tabulated by Puska 
TABLE I. Friction coefficients for a $\mathrm{C}$ atom as a function of the embedding mean electron radius $r_{s}$ as calculated with the LDA and LSDA (see Sec. II). All values in atomic units (a.u.).

\begin{tabular}{llllllllllll}
\hline \hline \multicolumn{10}{c}{$\eta$ (a.u.) } \\
\hline$r_{s}$ (a.u.) & 3.5 & 4.0 & 4.25 & 4.5 & 4.75 & 5.0 & 5.85 & 6.0 & 6.5 & 7.0 \\
LDA & 0.254 & 0.199 & 0.178 & 0.161 & 0.146 & 0.133 & $\ldots$ & 0.103 & $\ldots$ & $\ldots$ \\
LSDA & 0.254 & 0.166 & 0.127 & 0.1 & 0.077 & 0.061 & 0.031 & 0.028 & 0.022 & 0.018 \\
\hline \hline
\end{tabular}

and Nieminen for various atoms. ${ }^{83}$ The use of the local spin density approximation (LSDA) is obviously more appropriate, but the differences in the friction coefficient values for most atoms of interest are very minor and commonly neglected. An exception is the $\mathrm{C}$ atom at very low densities. Table I and Fig. 1 show the corresponding LDA and LSDA friction coefficient values as a function of the mean electron radius $r_{s}$, which is associated with the embedded electron density with $\rho=\frac{3}{4 \pi r_{s}^{3}}$. Note that the LDA and LSDA friction coefficients for $\mathrm{H}$ atoms are essentially the same. Since the differences already become apparent at densities that will be probed in our simulations $\left(r_{s}>4\right.$ a.u.), we opt for using the LSDA values when calculating the friction force on C. Nonetheless, we have also verified that the results of Section III were little changed if the LDA friction coefficients were used instead.
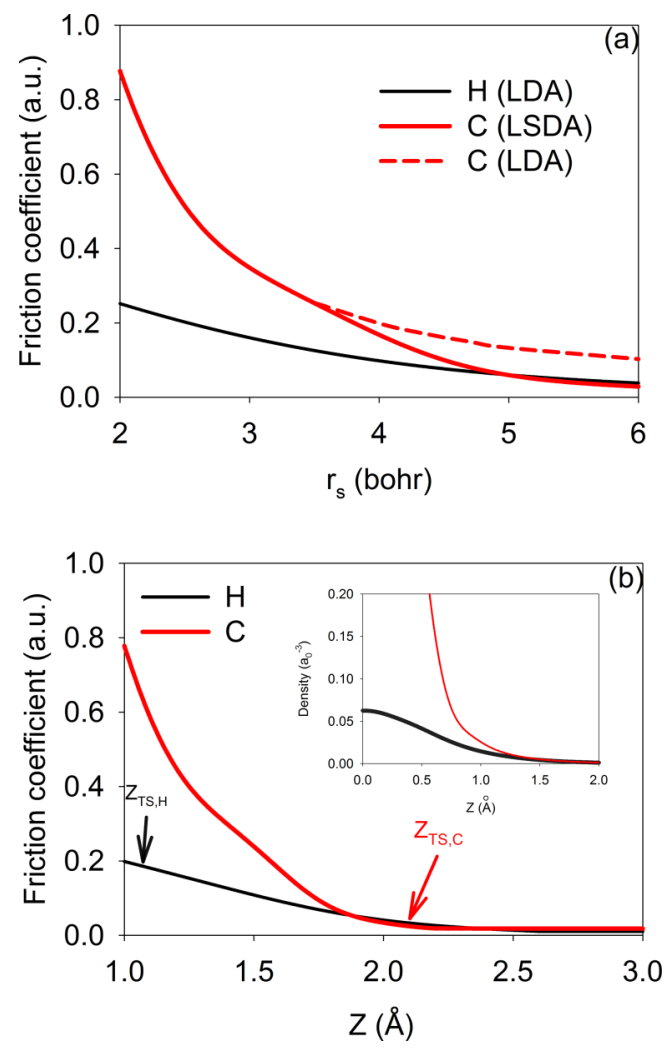

FIG. 1. (a) Friction coefficients of $\mathrm{H}$ and $\mathrm{C}$ as a function of the mean electron radius $r_{\mathrm{S}}$ defined as $r_{s}=\left(\frac{3}{4 \pi \rho}\right)^{1 / 3}$; both LSDA and LDA results for $\mathrm{C}$ atom are presented. (b) Friction coefficients for the $\mathrm{C}$ and dissociating $\mathrm{H}$ atoms in the molecule as a function of their vertical distances above the surface with the lateral coordinates fixed at the transition state $\left(Z_{\mathrm{TS}, \mathrm{C}}\right.$ and $Z_{\mathrm{TS}, \mathrm{H}}$ are marked by arrows), the corresponding electron densities over ( $\left.X_{\mathrm{TS}, \mathrm{C}}, Y_{\mathrm{TS}, \mathrm{C}}\right)$ and $\left(X_{\mathrm{TS}, \mathrm{H}}, Y_{\mathrm{TS}, \mathrm{H}}\right)$ as a function of the vertical distances are shown in the inset.
The use of an analytical PES in Eq. (1) offers substantially lower computational costs than the AIMD approach, enabling the calculations of many more trajectories to achieve good statistics, which are necessary to quantify the effects of EHP dissipation in various quantum states of $\mathrm{CH}_{4}$ and several of its partially deuterated isotopologues.

\section{Quasi-classical trajectory method}

The QCT calculations were performed with and without friction using a modified VENUS program. ${ }^{84}$ Since the $\mathrm{Ni}(111)$ surface is considered to be flat in our 12D PES, the embedded electron density for each atom was evaluated such that the center of mass $(X, Y)$ coordinates was fixed at the transition state. Standard normal mode sampling was applied to obtain the initial coordinates and momenta of rotationless $\mathrm{CH}_{4}$ and its deuterated isotopologues, followed by a random orientation. The translational energy ranges from 18 to $33 \mathrm{kcal} / \mathrm{mol}$ depending on the initial state. The integration time step was set to $0.10 \mathrm{fs}$, which conserves the total energy within $10^{-3} \mathrm{kcal} / \mathrm{mol}$ in the absence of friction. To control the standard error of the overall reactivity below $5 \%$, as many as $2 \times 10^{5}$ trajectories were run at the lowest translational energy. Initial separation between the molecule and the surface was $6.0 \AA$. The reactive trajectories are those for which the distance between the $\mathrm{C}$ atom and any $\mathrm{H}$ (or D) atom exceeded $2.2 \AA$, while the non-reactive ones are collected if the molecule reaches $6.1 \AA$ after being scattered off the surface. The dissociation probability was computed by the fraction of the number of reactive trajectories $\left(N_{r}\right)$ in total number of trajectories $\left(N_{\text {total }}\right)$.

\section{RESULTS AND DISCUSSION}

In Fig. 1(a), the computed friction coefficients for the $\mathrm{H}$ and $\mathrm{C}$ atoms according to Eq. (2) are compared as a function of the mean electron radius $r_{s}$. Note that $\eta_{C}$ is larger than $\eta_{H}$ when the electron density is high and both become negligible for very low densities. More explicitly, Fig. 1(b) displays the friction coefficients of $\mathrm{C}$ and $\mathrm{H}$ as a function of the vertical distance from the surface. Since our PES was constructed in the way that the lateral coordinates $(X, Y)$ of the molecular center are fixed at the transition state, the atomic coordinates are therefore subjected to this restriction. Both friction coefficients increase rapidly as the atoms move to the surface where the electron density is high. At the DC transition state, the dissociating $\mathrm{H}$ atom is closer to the surface $\left(Z_{\mathrm{H}}=1.09 \AA\right)$ than the $\mathrm{C}$ atom $\left(Z_{\mathrm{C}}=2.10 \AA\right)$, giving rise to larger $\eta_{H}$ than $\eta_{C}$. This phenomenon is similar to that in the DC of water on $\mathrm{Ni}(111)$.

Let us first discuss the dissociation of $\mathrm{CH}_{4}$. Figure 2(a) shows the QCT dissociation probabilities with and without friction for $\mathrm{CH}_{4}$ in its ground state and first excited state in each vibrational mode. Clearly, vibrational excitations strongly promote the reaction. More precisely, the symmetric $\left(v_{1}\right)$ and antisymmetric $\left(v_{3}\right)$ stretching modes show the comparably largest enhancements, followed by similar but smaller enhancements of the two bending modes $\left(v_{2}\right.$ and $v_{4}$ ). When EHP excitations are included, the dissociation 

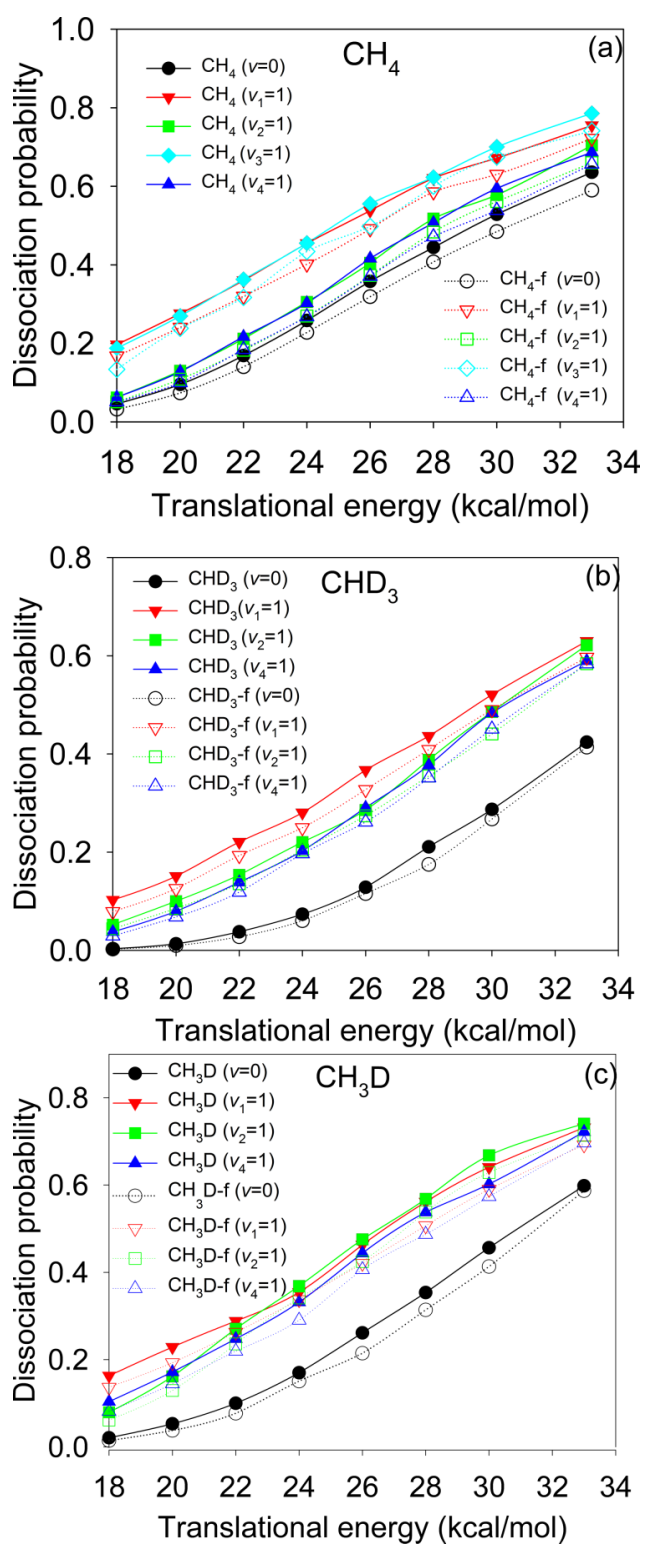

FIG. 2. Calculated overall reactivities for the $\mathrm{CH}_{4} / \mathrm{CHD}_{3} / \mathrm{CHD}_{3}$ dissociation on $\mathrm{Ni}(111)$ with (open symbols) and without friction (solid symbols) for various initial states.

probabilities generally decrease, which is consistent with previous observations in direct dissociation processes of $\mathrm{H}_{2}{ }^{54}$ and $\mathrm{H}_{2} \mathrm{O} .{ }^{59}$ This can be attributed to the competition between the dissociation and the EHP induced dissipation. ${ }^{59}$ However, the mode specificity is less affected by EHP excitations because the inhibition of the reactivity is similar for each $\mathrm{CH}_{4}$ vibrational state, as found in our previous study in the $\mathrm{D}_{2} \mathrm{O} / \mathrm{Ni}(111)$ system. $^{59}$

The vibrational efficacy for a vibrationally excited state with respect to the ground state is described by the ratio of the translational energy difference at a given dissociation probability $P_{0}$ between the two vibrational states and the corresponding vibrational excitation energy, i.e., $\eta_{v}$ $=\left[E_{n}\left(v, P_{0}\right)-E_{n}\left(v-1, P_{0}\right)\right] / \Delta E_{v}{ }^{85}$ Since the experimental data have been often fit to an $S$-shaped reactivity curve suggested by Luntz, ${ }^{85}$ with the same parameters of the widths and the saturation value of the reactivity for the ground and
TABLE II. The calculated vibrational efficacies with respect to overall reactivity of various vibrational modes in the dissociative chemisorption of $\mathrm{CH}_{4} / \mathrm{CHD}_{3} / \mathrm{CH}_{3} \mathrm{D}$ with and without electronic friction.

\begin{tabular}{llll}
\hline \hline & & \multicolumn{2}{c}{ Vibrational efficacy } \\
\cline { 3 - 4 } & Mode & Without friction & With friction \\
\hline & $v_{1}=1$ & $0.48-0.56$ & $0.46-0.59$ \\
$\mathrm{CH}_{4}$ & $v_{2}=1$ & $0.21-0.41$ & $0.21-0.51$ \\
& $v_{3}=1$ & $0.47-0.54$ & $0.45-0.59$ \\
& $v_{4}=1$ & $0.24-0.50$ & $0.24-0.45$ \\
\hline & $v_{1}=1$ & $0.47-0.69$ & $0.38-0.66$ \\
$\mathrm{CH}_{3} \mathrm{D}$ & $v_{2}=1$ & $0.60-0.70$ & $0.55-0.74$ \\
& $v_{4}=1$ & $0.38-0.47$ & $0.32-0.47$ \\
\hline & $v_{1}=1$ & $0.63-0.84$ & $0.57-0.79$ \\
$\mathrm{CHD}_{3}$ & $v_{2}=1$ & $0.66-0.85$ & $0.60-0.85$ \\
& $v_{4}=1$ & $0.59-0.68$ & $0.56-0.71$ \\
\hline \hline
\end{tabular}

excited state reaction probability curves, the corresponding vibrational efficacies are independent of translational energy. In practice, the calculated vibrational efficacy may depend sensitively on the translational energy. As listed in Table II, vibrational efficacies obtained with and without friction are found to vary in roughly the same ranges with small differences, given uncertainties in the estimate of the theoretical vibrational efficacies by interpolating the scattered QCT dissociation probabilities at specific energies.

Analogously, the overall reactivity of $\mathrm{CHD}_{3}$ and $\mathrm{CH}_{3} \mathrm{D}$, including both the $\mathrm{C}-\mathrm{H}$ and $\mathrm{C}-\mathrm{D}$ bond cleavages, is also presented in Figs. 2(b) and 2(c), with the molecules in the ground and first excited states in the $\mathrm{C}-\mathrm{H}$ or $\mathrm{C}-\mathrm{D}$ stretching modes, namely, $v_{1}, v_{2}$, and $v_{4}$, respectively. The vibrational energy in these excited states is expected to be highly localized in the vibrating bond(s), which would become reactive leading to prominent bond selective behavior. Note that the $v_{1}, v_{2}$, and $v_{4}$ modes represent the $\mathrm{CH}$ stretch, $\mathrm{CD}_{3}$ symmetric stretch, and $\mathrm{CD}_{3}$ antisymmetric stretch in $\mathrm{CHD}_{3}$, respectively, while they stand for the $\mathrm{CH}_{3}$ symmetric stretch, $\mathrm{CD}$ stretch, and $\mathrm{CH}_{3}$ antisymmetric stretch in $\mathrm{CH}_{3} \mathrm{D}$. It is found that $\mathrm{CHD}_{3}\left(v_{1}=1\right)$ is in general more reactive than $\mathrm{CHD}_{3}\left(v_{2}=1\right)$ and $\mathrm{CHD}_{3}\left(v_{4}=1\right)$ in the energy range studied here. On the other hand, $\mathrm{CH}_{3} \mathrm{D}\left(v_{1}=1\right)$ shows slightly higher enhancement at low energies over the other two, while their reaction probabilities are close to each other at high energies. Similar to the $\mathrm{CH}_{4}$ case, EHP excitations, described by the electronic friction, appear to suppress the dissociation of all initial states, to roughly the same extent. As a result, the vibrational efficacies do not change significantly, as given in Table II.

Interestingly, all QCT vibrational efficacies are found to be much smaller than experimental values ${ }^{4}$ and reduced dimensional quantum results, ${ }^{30,31}$ despite the fact that they reasonably reproduce the general trend, e.g., the stretching modes are generally more efficacious than the bending ones. Since our QCT calculations are based on the flat surface model and the full dimensional QD calculations are not yet available, we defer a quantitative comparison to the future work. In the case of $\mathrm{H}_{2} \mathrm{O}$ dissociation on $\mathrm{Ni}(111)$, the full- 
dimensional static QCT predicted vibrational efficacies are only slightly smaller than their quantum counterparts and all exceed unity. ${ }^{59,86}$

While the overall vibrational efficacies are similar, the specific vibrational efficacies for breaking the $\mathrm{C}-\mathrm{H}$ and $\mathrm{C}-\mathrm{D}$ bonds are quite different. The so-called bond selectivity has been observed in experiments ${ }^{14,17,19,23}$ and interpreted using various models. ${ }^{32,33,35,45}$ Here, the influence of EHPs on bond selectivity is investigated using the same friction approach, in which the same friction coefficient is used for $\mathrm{H}$ and D. The calculated reaction probabilities for $\mathrm{C}-\mathrm{H}$ and $\mathrm{C}-\mathrm{D}$ dissociation channels of $\mathrm{CHD}_{3}$ and $\mathrm{CH}_{3} \mathrm{D}$ with and without friction are presented in Figs. 3 and 4, respectively. The calculated branching ratios are also listed in Table III. For $\mathrm{CHD}_{3}$, as found in previous studies, ${ }^{32,35,45}$ the excitation of the $\mathrm{C}-\mathrm{H}$ stretching $\left(v_{1}\right)$ mode substantially promotes the cleavage of $\mathrm{C}-\mathrm{H}$ bond but has a negligible effect on the C-D bond breaking, while the $\mathrm{CD}_{3}$ stretching $\left(v_{2}\right.$ and $\left.v_{4}\right)$ excited states preferentially break the $\mathrm{C}-\mathrm{D}$ bond with comparable reactivity but have little influence on the $\mathrm{H}+\mathrm{CD}_{3}$ product channel. In the case of $\mathrm{CH}_{3} \mathrm{D}$, as expected, the localized $\mathrm{C}-\mathrm{D}$ stretching excited state $\left(v_{2}=1\right)$ strongly enhances the $\mathrm{C}-\mathrm{D}$ cleavage channel but has a minor effect on the $\mathrm{C}-\mathrm{H}$ cleavage channel. While $\mathrm{CH}_{3} \mathrm{D}\left(v_{1}=1\right)$ and $\mathrm{CH}_{3} \mathrm{D}\left(v_{4}=1\right)$ barely promote the $\mathrm{C}-\mathrm{D}$ cleavage channel, they show enhancement to yield the $\mathrm{H}+\mathrm{CH}_{2} \mathrm{D}$ products, breaking the $\mathrm{C}-\mathrm{H}$ bond. Interestingly, this bond selectivity in $\mathrm{CHD}_{3} / \mathrm{CH}_{3} \mathrm{D}$ dissociation becomes less pronounced as the translational energy increases. As suggested by Shen et al. ${ }^{35}$ the loss of bond selectivity can be attributed to the fact that the increasing translational energy itself favors
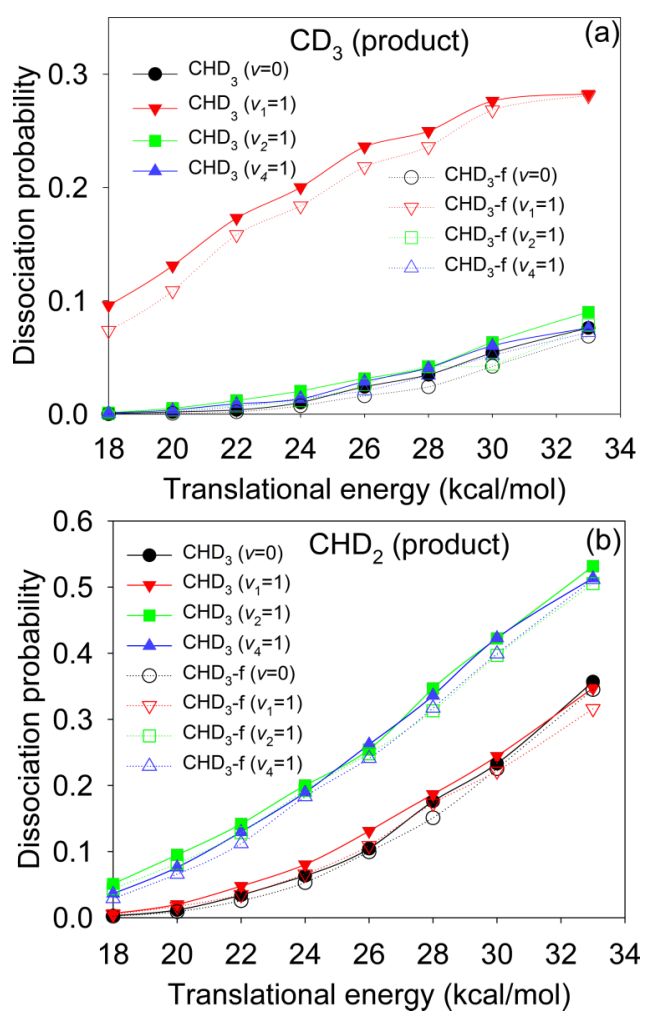

FIG. 3. Calculated bond selective dissociation probabilities of $\mathrm{CHD}_{3}$ leading to $\mathrm{CD}_{3}$ (top panel) and $\mathrm{CHD}_{2}$ products (bottom panel) on $\mathrm{Ni}(111)$ with (open symbols) and without friction (solid symbols) for various initial states.
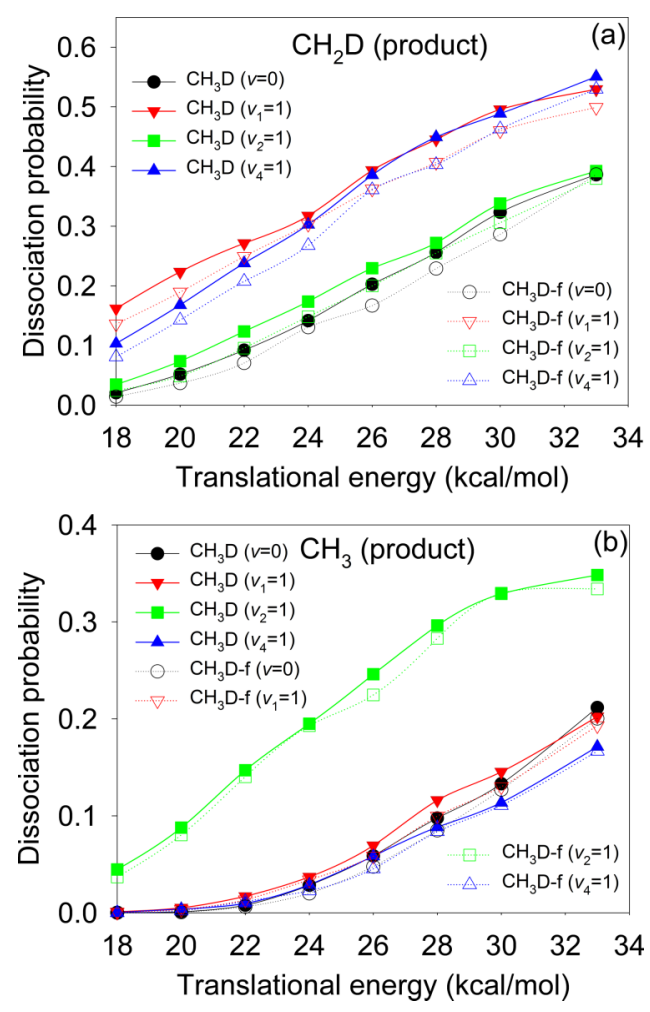

FIG. 4. Calculated bond selective dissociation probabilities of $\mathrm{CH}_{3} \mathrm{D}$ leading to $\mathrm{CH}_{2} \mathrm{D}$ (top panel) and $\mathrm{CH}_{3}$ products (bottom panel) on $\mathrm{Ni}(111)$ with (open symbols) and without friction (solid symbols) for various initial states.

more non-selectively reactive events, thus weakening the role of the initial vibrational energy in inducing bond selectivity.

The electronic friction uniformly decreases the bond selective reactivity for all initial states to a similar level, with the $\mathrm{C}-\mathrm{H}$ bond cleavage being slightly more inhibited than the $\mathrm{C}-\mathrm{D}$ bond cleavage when the corresponding bond is pre-excited, which may be attributed to higher $\mathrm{C}-\mathrm{H}$ stretching velocity than the $\mathrm{C}-\mathrm{D}$ one. However, the branching ratios are not altered in a significant way. The seemingly large numerical difference in some low energy results with and without friction, e.g., at $18 \mathrm{kcal} / \mathrm{mol}$ for $\mathrm{CH}_{3} \mathrm{D}\left(v_{1}=1\right)$ and $\mathrm{CH}_{3} \mathrm{D}\left(v_{4}=1\right)$, is due presumably to the very small C-D dissociation probability making the branching ratio sensitive to the slight perturbation in bond selective reactivity. However, it is clear that the near full selectivity of the $\mathrm{C}-\mathrm{H}$ bond cleavage in $\mathrm{CH}_{3} \mathrm{D}\left(v_{1}=1\right)$ or $\mathrm{CH}_{3} \mathrm{D}\left(v_{4}=1\right)$ is achieved regardless of the EHP effects. These results are in general accord with experiments of Beck et al. on $\mathrm{Pt}(111)^{19,33}$ and Utz et al. on $\mathrm{Ni}(111) .{ }^{14}$ In addition, a single quantum excited in $v_{2}$ mode of $\mathrm{CH}_{3} \mathrm{D}$ does not lead to full selectivity of C-D bond cleavage, as observed by Shen et al. ${ }^{35}$ who have found that the two-quanta excitation of $v_{2}$ is needed to completely break the C-D bond.

To gain further insight into the EHP effects on mode specificity, we plot energy loss distributions for the DC of $\mathrm{CH}_{4}$ in Fig. 5. As can be seen in Fig. 5(a), for the ground state $\mathrm{CH}_{4}$, the distribution of energy loss to EHPs in reactive trajectories varies only slightly as the translational energy increases. This is presumably because molecular-surface interaction time decreases when atomic velocities increase, thus keeping the energy loss little changed. On the other hand, the energy 
TABLE III. Calculated branching ratios of products by $\mathrm{C}-\mathrm{H}$ or $\mathrm{C}-\mathrm{D}$ bond cleavage in $\mathrm{CHD}_{3} / \mathrm{CH}_{3} \mathrm{D}$ dissociation on $\mathrm{Ni}(111)$.

\begin{tabular}{|c|c|c|c|c|c|c|}
\hline \multirow[b]{2}{*}{$\mathrm{E}_{\text {trans }}(\mathrm{kcal} / \mathrm{mol})$} & \multirow[b]{2}{*}{ Species } & \multicolumn{2}{|c|}{$\mathrm{CH}_{3} / \mathrm{CH}_{2} \mathrm{D}$ branching ratio } & \multirow[b]{2}{*}{ Species } & \multicolumn{2}{|c|}{$\mathrm{CD}_{3} / \mathrm{CHD}_{2}$ branching ratio } \\
\hline & & Without friction & With friction & & Without friction & With friction \\
\hline 18 & $\mathrm{CH}_{3} \mathrm{D}\left(v_{1}=1\right)$ & 0.00695 & 0.00408 & $\mathrm{CHD}_{3}\left(v_{1}=1\right)$ & 14.5 & 14.3 \\
\hline 20 & & 0.0228 & 0.0179 & & 6.51 & 6.40 \\
\hline 22 & & 0.0627 & 0.0535 & & 3.63 & 4.57 \\
\hline 24 & & 0.117 & 0.112 & & 2.49 & 2.78 \\
\hline 26 & & 0.176 & 0.161 & & 1.80 & 2.00 \\
\hline 28 & & 0.260 & 0.245 & & 1.34 & 1.36 \\
\hline 30 & & 0.293 & 0.283 & & 1.13 & 1.21 \\
\hline 33 & & 0.382 & 0.387 & & 0.813 & 0.889 \\
\hline 18 & $\mathrm{CH}_{3} \mathrm{D}\left(v_{2}=1\right)$ & 1.29 & 1.50 & $\mathrm{CHD}_{3}\left(v_{2}=1\right)$ & 0.0246 & 0.00929 \\
\hline 20 & & 1.19 & 1.64 & & 0.0537 & 0.0427 \\
\hline 22 & & 1.19 & 1.47 & & 0.0847 & 0.0560 \\
\hline 24 & & 1.12 & 1.30 & & 0.102 & 0.0610 \\
\hline 26 & & 1.07 & 1.12 & & 0.123 & 0.105 \\
\hline 28 & & 1.09 & 1.11 & & 0.119 & 0.134 \\
\hline 30 & & 0.973 & 1.08 & & 0.150 & 0.111 \\
\hline 33 & & 0.888 & 0.879 & & 0.169 & 0.156 \\
\hline 18 & $\mathrm{CH}_{3} \mathrm{D}\left(v_{4}=1\right)$ & 0.00351 & 0.00176 & $\mathrm{CHD}_{3}\left(v_{4}=1\right)$ & 0.0308 & 0.0152 \\
\hline 20 & & 0.0226 & 0.0140 & & 0.0460 & 0.0334 \\
\hline 22 & & 0.0406 & 0.0562 & & 0.0694 & 0.0585 \\
\hline 24 & & 0.0958 & 0.0847 & & 0.0704 & 0.0747 \\
\hline 26 & & 0.151 & 0.126 & & 0.109 & 0.0849 \\
\hline 28 & & 0.197 & 0.207 & & 0.121 & 0.108 \\
\hline 30 & & 0.233 & 0.239 & & 0.143 & 0.129 \\
\hline 33 & & 0.311 & 0.315 & & 0.148 & 0.141 \\
\hline
\end{tabular}

loss distributions for different vibrational states of $\mathrm{CH}_{4}$ at a given incident energy are also quite similar, as shown in Fig. 5(b), consistent with the marginal effect on the mode specificity. It should be noted that the energy loss due to the electronic friction in this case is on average of $\sim 2 \mathrm{kcal} / \mathrm{mol}$, which is somewhat higher than that in $\mathrm{H}_{2} \mathrm{O} / \mathrm{Ni}(111)$ system (on average of $\sim 1 \mathrm{kcal} / \mathrm{mol}$ ), ${ }^{59}$ probably because methane contains more atoms, each of which dissipates some energy. However, it is still quite small and insufficient for changing the direct reaction mechanism in the methane DC. As a result, we conclude that EHP effects are still relatively minor in direct DC processes.

Very recently, a more accurate specific reaction parameter density functional has recently been proposed by Nattino $e t a l$. for $\mathrm{CHD}_{3} / \mathrm{Ni}(111),{ }^{87}$ which actually yields a barrier height of $23.4 \mathrm{kcal} / \mathrm{mol}, \sim 1.7 \mathrm{kcal} / \mathrm{mol}$ (or $7 \%$ ) lower than that in the PES used here. The small change of barrier height should only change the relative energy (velocity) over the transition state at a given translational energy, but not the trend of the EHP effects at a series of translational energies. Since we investigate the EHP effects as a function of translational energy and focus on the vibrational efficacies, this would not alter our conclusion.

\section{SUMMARY}

In the present work, we report an extensive examination of the surface EHP effects on the DC of methane on rigid
$\mathrm{Ni}(111)$ using the LDFA-IAA model implemented with a QCT method. The use of our recently developed PES with 12 DOFs allowed us to examine the EHP effects in various isotopologues of methane in several vibrational states. The excitations of surface EHPs are effectively treated as electronic friction forces proportional to the velocity of the constituent atoms. It is found that the energy dissipation due to EHP excitations is generally small, similar to the case of water dissociation on $\mathrm{Ni}(111)$, which leads to a minor decrease of the dissociation probability for each vibrational state of $\mathrm{CH}_{4}$ and its isotopologues. This inhibition is similar for most vibrational states resulting in $20 \%$ variations at most in the vibrational efficacies, as a result, the EHPs have a limited impact on the mode specificity and bond selectivity in dissociative chemisorption within the LDFA-IAA framework.

We note that our model still contains many approximations. Despite the fact that the LDFA-IAA approximation has previously proved to be reasonable, this treatment completely ignores the intramolecular contributions to the friction coefficients. In this respect, Rittmeyer et al. ${ }^{81}$ recently proposed a model based on the LDFA that included the intramolecular effects based on the Hirshfeld charge partitioning scheme. ${ }^{88}$ The method effectively treats the molecular electrons as part of the metallic substrate that in principle can be well justified for chemisorbed adsorbates at close distance to the surface and, therefore, it is expected to account better for the vibrational damping rates of these adsorbates' modes. However, the method is not applicable to 

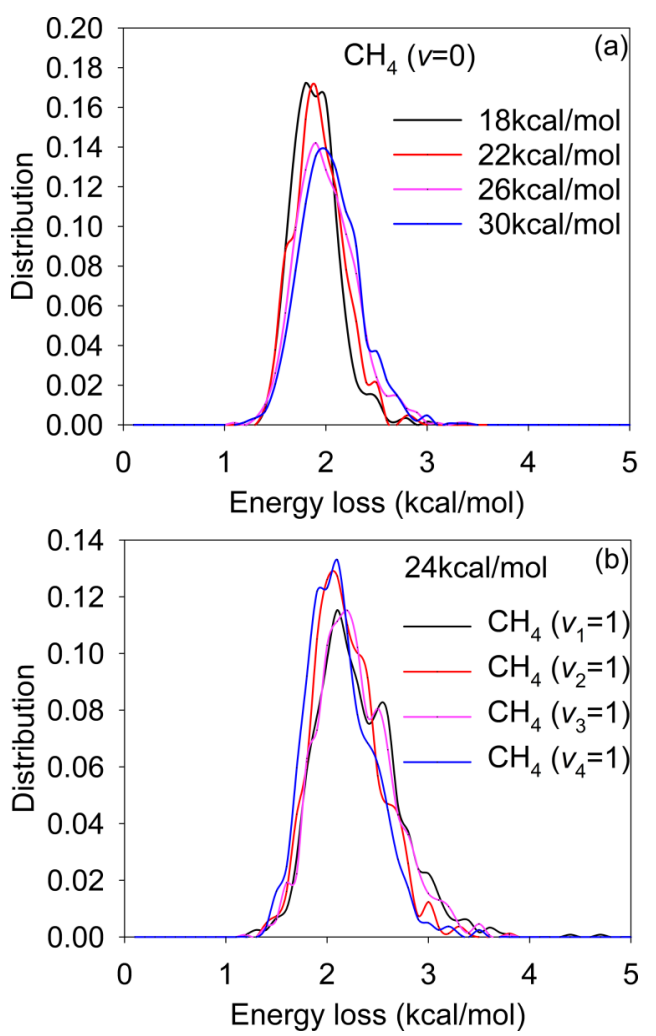

FIG. 5. (a) Normalized distributions of energy loss to EHPs in the ground state $\mathrm{CH}_{4}$ dissociation process with different incident energies. (b) The same as (a) except for $\mathrm{CH}_{4}$ in its ground and vibrationally excited states at an incident energy of $24 \mathrm{kcal} / \mathrm{mol}$.

scattering experiments as the ones treated here, in which large molecule surface distances are involved, because it will give rise to unphysical energy dissipation effects. Therefore, further theoretical research along these lines as well as on obtaining the friction tensor ${ }^{89}$ that incorporates the different degrees of freedom of polyatomic molecules is highly desirable. In addition, the current calculations neglect the influence of site dependence and surface phonons, because of the PES used in the calculations. Although the former may not be essential, the later could provide the major dissipation channel in DC process and compete with the EHP excitation channel. ${ }^{71,75}$ How these issues affect the quantitative treatment of the EHP effects in DC of polyatomic molecules remains an open question. Nevertheless, it is our belief that mode specificity and bond selectivity, which are largely determined by the direct nature of the dissociation process, are unlikely to be strongly affected by EHPs because of the short interaction time between the molecule and metal electrons.

\section{ACKNOWLEDGMENTS}

We thank the National Natural Science Foundation of China (Grant No. 21573203 to B.J.) and the U.S. National Science Foundation (Grant No. CHE-1462019 to H.G.). J.I.J. and M.A. acknowledge financial support by the Spanish Ministerio de Economía y Competitividad (Grant No. FIS2013-48286-C2-2-P). Calculations have been done on the supercomputing system in the Supercomputing Center of University of Science and Technology of China.
${ }^{1}$ J. R. Rostrup-Nielsen, in Catalysis, Science and Technology, edited by J. R. Anderson and M. Boudart (Springer-Verlag, Berlin, 1984), Vol. 5.

${ }^{2}$ L. B. F. Juurlink, D. R. Killelea, and A. L. Utz, Prog. Surf. Sci. 84, 69 (2009),

${ }^{3}$ G.-J. Kroes, Phys. Chem. Chem. Phys. 14, 14966 (2012).

${ }^{4}$ R. D. Beck and A. L. Utz, in Dynamics of Gas-Surface Interactions, edited by R. D. Muiño and H. F. Busnengo (Springer, Heidelberg, 2013).

${ }^{5}$ S. Nave, A. K. Tiwari, and B. Jackson, J. Phys. Chem. A 118, 9615 (2014).

${ }^{6}$ B. Jiang, M. Yang, D. Xie, and H. Guo, Chem. Soc. Rev. 45, 3621 (2016).

${ }^{7}$ L. B. F. Juurlink, P. R. MaCabe, R. R. Smith, C. L. DeCologero, and A. L. Utz, Phys. Rev. Lett. 83, 868 (1999).

${ }^{8}$ M. P. Schmid, P. Maroni, R. D. Beck, and T. R. Rizzo, J. Chem. Phys. 117, 8603 (2002).

${ }^{9}$ R. D. Beck, P. Maroni, D. C. Papageorgopoulos, T. T. Dang, M. P. Schmid, and T. R. Rizzo, Science 302, 98 (2003).

${ }^{10}$ R. R. Smith, D. R. Killelea, D. F. DelSesto, and A. L. Utz, Science 304, 992 (2004).

${ }^{11}$ P. Maroni, D. C. Papageorgopoulos, M. Sacchi, T. T. Dang, R. D. Beck, and T. R. Rizzo, Phys. Rev. Lett. 94, 246104 (2005).

${ }^{12}$ L. B. F. Juurlink, R. R. Smith, D. R. Killelea, and A. L. Utz, Phys. Rev. Lett. 94, 208303 (2005).

${ }^{13}$ R. Bisson, M. Sacchi, T. T. Dang, B. Yoder, P. Maroni, and R. D. Beck, J. Phys. Chem. A 111, 12679 (2007).

${ }^{14}$ D. R. Killelea, V. L. Campbell, N. S. Shuman, and A. L. Utz, Science 319, 790 (2008).

${ }^{15}$ D. R. Killelea, V. L. Campbell, N. S. Shuman, and A. L. Utz, J. Phys. Chem. C 113, 20618 (2009).

${ }^{16}$ R. Bisson, M. Sacchi, and R. D. Beck, J. Chem. Phys. 132, 094702 (2010).

${ }^{17}$ B. L. Yoder, R. Bisson, and R. D. Beck, Science 329, 553 (2010).

${ }^{18}$ B. L. Yoder, R. Bisson, P. M. Hundt, and R. D. Beck, J. Chem. Phys. 135, 224703 (2011).

${ }^{19}$ L. Chen, H. Ueta, R. Bisson, and R. D. Beck, Faraday Discuss. 157, 285 (2012).

${ }^{20}$ N. Chen, Y. Huang, and A. L. Utz, J. Phys. Chem. A 117, 6250 (2013).

${ }^{21}$ P. M. Hundt, M. E. van Reijzen, H. Ueta, and R. D. Beck, J. Phys. Chem. Lett. 5, 1963 (2014).

${ }^{22}$ E. Dombrowski, E. Peterson, D. Del Sesto, and A. L. Utz, Catal. Today 244, 10 (2015).

${ }^{23}$ P. M. Hundt, H. Ueta, M. E. van Reijzen, B. Jiang, H. Guo, and R. D. Beck, J. Phys. Chem. A 119, 12442 (2015).

${ }^{24}$ P. M. Hundt, B. Jiang, M. van Reijzen, H. Guo, and R. D. Beck, Science 344, 504 (2014).

${ }^{25}$ S. Nave and B. Jackson, Phys. Rev. Lett. 98, 173003 (2007).

${ }^{26}$ A. K. Tiwari, S. Nave, and B. Jackson, Phys. Rev. Lett. 103, 253201 (2009).

${ }^{27}$ G. P. Krishnamohan, R. A. Olsen, G.-J. Kroes, F. Gatti, and S. Woittequand, J. Chem. Phys. 133, 144308 (2010).

${ }^{28}$ A. K. Tiwari, S. Nave, and B. Jackson, J. Chem. Phys. 132, 134702 (2010).

${ }^{29}$ B. Jackson and S. Nave, J. Chem. Phys. 135, 114701 (2011).

${ }^{30}$ B. Jackson and S. Nave, J. Chem. Phys. 138, 174705 (2013).

${ }^{31}$ B. Jiang, R. Liu, J. Li, D. Xie, M. Yang, and H. Guo, Chem. Sci. 4, 3249 (2013).

${ }^{32}$ B. Jiang and H. Guo, J. Phys. Chem. C 117, 16127 (2013).

${ }^{33}$ F. Nattino, H. Ueta, H. Chadwick, M. E. van Reijzen, R. D. Beck, B. Jackson, M. C. van Hemert, and G.-J. Kroes, J. Phys. Chem. Lett. 5, 1294 (2014).

${ }^{34}$ B. Jackson, F. Nattino, and G.-J. Kroes, J. Chem. Phys. 141, 054102 (2014).

${ }^{35}$ X. J. Shen, A. Lozano, W. Dong, H. F. Busnengo, and X. H. Yan, Phys. Rev. Lett. 112, 046101 (2014).

${ }^{36}$ A. Lozano, X. J. Shen, R. Moiraghi, W. Dong, and H. F. Busnengo, Surf. Sci. 640, 25 (2015).

${ }^{37}$ X. Shen, J. Chen, Z. Zhang, K. Shao, and D. H. Zhang, J. Chem. Phys. 143, 144701 (2015).

${ }^{38}$ X. Shen, Z. Zhang, and D. H. Zhang, Phys. Chem. Chem. Phys. 17, 25499 (2015).

${ }^{39}$ F. Nattino, D. Migliorini, M. Bonfanti, and G.-J. Kroes, J. Chem. Phys. 144, 044702 (2016).

${ }^{40}$ X. Shen, Z. Zhang, and D. H. Zhang, J. Chem. Phys. 144, 101101 (2016).

${ }^{41}$ G. Füchsel, P. S. Thomas, J. den Uyl, Y. Ozturk, F. Nattino, H.-D. Meyer, and G.-J. Kroes, Phys. Chem. Chem. Phys. 18, 8174 (2016).

${ }^{42}$ B. Jiang and H. Guo, J. Phys. Chem. C 120, 8220 (2016).

${ }^{43}$ W. H. Miller, N. C. Handy, and J. E. Adams, J. Chem. Phys. 72, 99 (1980).

${ }^{44}$ M. Mastromatteo and B. Jackson, J. Chem. Phys. 139, 194701 (2013).

${ }^{45}$ H. Guo and B. Jackson, J. Phys. Chem. C 119, 14769 (2015).

${ }^{46}$ B. Jiang, D. Xie, and H. Guo, Chem. Sci. 4, 503 (2013).

${ }^{47} \mathrm{H}$. Guo and B. Jiang, Acc. Chem. Res. 47, 3679 (2014).

${ }^{48}$ J. C. Tully, Annu. Rev. Phys. Chem. 51, 153 (2000).

${ }^{49}$ H. Guo, P. Saalfrank, and T. Seideman, Prog. Surf. Sci. 62, 239 (1999). 
${ }^{50} \mathrm{H}$. Nienhaus, Surf. Sci. Rep. 45, 1 (2002).

${ }^{51}$ P. Saalfrank, G. Fuchsel, S. Monturet, J. C. Tremblay, and T. Klamroth, in Dynamics of Gas-Surface Interactions, edited by R. D. Muiño and H. F. Busnengo (Springer, Heidelberg, 2013).

${ }^{52}$ K. Golibrzuch, N. Bartels, D. J. Auerbach, and A. M. Wodtke, Annu. Rev. Phys. Chem. 66, 399 (2015).

${ }^{53}$ G. Füchsel, J. C. Tremblay, and P. Saalfrank, J. Chem. Phys. 141, 094704 (2014).

${ }^{54}$ J. I. Juaristi, M. Alducin, R. Díez Muiño, H. F. Busnengo, and A. Salin, Phys. Rev. Lett. 100, 116102 (2008).

${ }^{55}$ I. Goikoetxea, J. I. Juaristi, M. Alducin, and R. Diez Muiño, J. Phys.: Condens. Matter 21, 264007 (2009).

${ }^{56}$ P. Nieto, E. Pijper, D. Barredo, G. Laurent, R. Olsen, E. J. Baerends, G.-J. Kroes, and D. Farias, Science 312, 86 (2006).

${ }^{57}$ C. Diaz, J. K. Vincent, G. P. Krishnamohan, R. A. Olsen, G. J. Kroes, K. Honkala, and J. K. Norskov, J. Chem. Phys. 125, 114706 (2006).

${ }^{58}$ A. C. Luntz and M. Persson, J. Chem. Phys. 123, 074704 (2005).

${ }^{59}$ B. Jiang, M. Alducin, and H. Guo, J. Phys. Chem. Lett. 7, 327 (2016).

${ }^{60}$ J. P. Perdew, J. A. Chevary, S. H. Vosko, K. A. Jackson, M. R. Pederson, D. J. Singh, and C. Fiolhais, Phys. Rev. B 46, 6671 (1992).

${ }^{61}$ B. J. Braams and J. M. Bowman, Int. Rev. Phys. Chem. 28, 577 (2009).

${ }^{62}$ B. Jiang, X. Ren, D. Xie, and H. Guo, Proc. Natl. Acad. Sci. U. S. A. 109, 10224 (2012).

${ }^{63}$ Y. Li and G. Wahnström, Phys. Rev. Lett. 68, 3444 (1992).

${ }^{64}$ M. Head-Gordon and J. C. Tully, J. Chem. Phys. 103, 10137 (1995).

${ }^{65}$ J. C. Tully, J. Chem. Phys. 73, 1975 (1980).

${ }^{66}$ P. M. Echenique, R. M. Nieminen, and R. H. Ritchie, Solid State Commun. 37, 779 (1981).

${ }^{67}$ P. M. Echenique, R. M. Nieminen, J. C. Ashley, and R. H. Ritchie, Phys. Rev. A 33, 897 (1986).

${ }^{68}$ J. C. Tremblay and P. Saalfrank, J. Chem. Phys. 131, 084716 (2009).

${ }^{69}$ J. Meyer and K. Reuter, New J. Phys. 13, 085010 (2011).
${ }^{70}$ A. S. Muzas, J. I. Juaristi, M. Alducin, R. D. Muiño, G. J. Kroes, and C. Díaz, J. Chem. Phys. 137, 064707 (2012).

${ }^{71}$ L. Martin-Gondre, M. Alducin, G. A. Bocan, R. Díez Muiño, and J. I. Juaristi, Phys. Rev. Lett. 108, 096101 (2012).

${ }^{72}$ M. Blanco-Rey, J. I. Juaristi, R. Díez Muiño, H. F. Busnengo, G. J. Kroes, and M. Alducin, Phys. Rev. Lett. 112, 103203 (2014).

${ }^{73}$ G.-J. Kroes, M. Pavanello, M. Blanco-Rey, M. Alducin, and D. J. Auerbach, J. Chem. Phys. 141, 054705 (2014).

${ }^{74}$ S. M. Janke, D. J. Auerbach, A. M. Wodtke, and A. Kandratsenka, J. Chem. Phys. 143, 124708 (2015).

${ }^{75}$ D. Novko, M. Blanco-Rey, J. I. Juaristi, and M. Alducin, Phys. Rev. B 92, 201411(R) (2015)

${ }^{76}$ O. Bünermann, H. Jiang, Y. Dorenkamp, A. Kandratsenka, S. M. Janke, D. J. Auerbach, and A. M. Wodtke, Science 350, 1346 (2015).

${ }^{77}$ M. Persson and B. Hellsing, Phys. Rev. Lett. 49, 662 (1982).

${ }^{78}$ B. Hellsing and M. Persson, Phys. Scr. 29, 360 (1984).

${ }^{79}$ M. Head-Gordon and J. C. Tully, J. Chem. Phys. 96, 3939 (1992).

${ }^{80}$ G. Füchsel, T. Klamroth, S. Monturet, and P. Saalfrank, Phys. Chem. Chem. Phys. 13, 8659 (2011)

${ }^{81}$ S. P. Rittmeyer, J. Meyer, J. I. Juaristi, and K. Reuter, Phys. Rev. Lett. 115, 046102 (2015).

${ }^{82}$ G. Füchsel, S. Schimka, and P. Saalfrank, J. Phys. Chem. A 117, 8761 (2013).

${ }^{83}$ M. J. Puska and R. M. Nieminen, Phys. Rev. B 27, 6121 (1983).

${ }^{84}$ X. Hu, W. L. Hase, and T. Pirraglia, J. Comput. Chem. 12, 1014 (1991).

${ }^{85}$ A. C. Luntz, J. Chem. Phys. 113, 6901 (2000).

${ }^{86}$ B. Jiang, H. Song, M. Yang, and H. Guo, J. Chem. Phys. 144, 164706 (2016).

${ }^{87}$ F. Nattino, D. Migliorini, G.-J. Kroes, E. Dombrowski, E. A. High, D. R. Killelea, and A. L. Utz, J. Phys. Chem. Lett. 7, 2402 (2016).

${ }^{88}$ F. L. Hirshfeld, Theor. Chim. Acta 44, 129 (1977).

${ }^{89}$ M. Askerka, R. J. Maurer, V. S. Batista, and J. C. Tully, Phys. Rev. Lett. 116, 217601 (2016). 